\title{
Tolerância de duas variedades de algodoeiro a herbicidas aplicados em pré- emergência ${ }^{1}$
}

\section{Tolerance of two cotton varieties to herbicides applied in pre-emergence}

João Guilherme Zanetti de Arantes ${ }^{2}$; Rubem Silvério de Oliveira Jr. ${ }^{3}$; Jamil Constantin ${ }^{3}$; Denis Fernando Biffe ${ }^{3}$; Michel Alex Raimondi ${ }^{4}$; Eder Blainski ${ }^{5}$; Diego Gonçalves Alonso ${ }^{4}$; Guilherme Braga Pereira Braz ${ }^{3}$

Resumo - Este trabalho teve por objetivo avaliar a seletividade de herbicidas aplicados em préemergência, isolados ou em misturas, para as variedades Delta-Opal e FMT-701. Os tratamentos avaliados foram: alachlor, S-metolachlor, diuron (em duas doses), prometryn (em duas doses), oxyfluorfen, alachlor + diuron, alachlor + prometryn (em duas doses da mistura), S-metolachlor + diuron, S-metolachlor + prometryn (em duas doses da mistura), oxyfluorfen + diuron, oxyfluorfen + prometryn. Foram realizadas avaliações relacionadas à fitointoxicação visual, altura de plantas e produtividade da cultura. Quando houve incremento na dose de diuron e prometryn, os sintomas de fitointoxicação foram intensificados em relação às menores doses. $\mathrm{O}$ herbicida oxyfluorfen quando aplicado isolado e em mistura com diuron ou prometryn proporcionou sintomas intensos de fitointoxicação à cultura do algodoeiro. No entanto, o único tratamento que não foi seletivo para ambas variedades de algodoeiro foi a mistura oxyfluorfen + prometryn $\left(0,192+0,900 \mathrm{~kg} \mathrm{ha}^{-1}\right)$.

Palavras-chaves: Gossypium hirsutum, mistura em tanque, pré-emergentes, seletividade

\begin{abstract}
This work aimed to evaluate the selectivity of herbicides applied pre-emergence, isolated or in tank-mixtures, for two cotton varieties, Delta-Opal and FMT-701. Treatments applied were: alachlor, S-metolachlor, diuron (two rates), prometryn (two rates), oxyfluorfen, alachlor + diuron, alachlor + prometryn (two rates of this mixture), S-metolachlor + diuron, Smetolachlor + prometryn (two rates of this mixture), oxyfluorfen + diuron, oxyfluorfen + prometryn. Evaluations performed were related to the visual plant intoxication, height of plants and crop yield. When rates of diuron and prometryn were increased, symptoms of crop injury were also more evident, in relation to lower rates of the same products. Oxyfluorfen, isolated or in tank mixture with diuron or prometryn propitiated intense symptoms of crop injury, but the only treatment which was considered as non-selective for both cotton varieties was the mixture of oxyfluorfen + prometryn $\left(0.192+0.9 \mathrm{~kg} \mathrm{ha}^{-1}\right)$.
\end{abstract}

Keywords: Gossypium hirsutum, tank mixture, pre-emergence, selectivity

\footnotetext{
${ }^{1}$ Recebido para publicação em 03/06/2014 e aceito em 15/04/2015.

2 Docente do curso de Agronomia da Universidade de Cuiabá (UNIC), Primavera do Leste-MT, Brasil. arantesjgz@yahoo.com.br (*autor para correspondência)

${ }^{3}$ Universidade Estadual de Maringá (UEM/NAPD), Maringá-PR, Brasil.

${ }^{4}$ Engenheiro Agrônomo, Doutor.

${ }^{5}$ Engenheiro Agrônomo, Mestre.
} 


\section{Introdução}

Após ter mais de três milhões de hectares cultivados no Centro-sul e no Nordeste do Brasil nos anos 60 e 70 e se caracterizar por uma cultura de pequenas áreas e baixa tecnologia, o algodoeiro praticamente não resistiu à entrada do bicudo-do-algodoeiro. Juntamente a isso, o elevado custo de mão-deobra e a abertura de novas fronteiras comerciais brasileiras, provocou uma inversão na cotonicultura do país, onde, de grande exportador, passou a ser o maior importador do mundo na década de 90 (Constantin et al., 2007). Com o desenvolvimento da soja e do milho nas regiões Centro-oeste e Nordeste, a cotonicultura migrou principalmente para estas áreas, como alternativa de rotação de culturas, mudando o cenário de produção sob o qual era explorada inicialmente no país, tornando-se uma cultura extremamente tecnificada.

Entre os fatores que afetam a produção desta cultura, cita-se a interferência imposta pela comunidade infestante, sendo o algodoeiro extremamente sensível aos danos causados pelas plantas daninhas. Esta sensibilidade está relacionada a alguns fatores morfofisiológicos e fitotécnicos como metabolismo fotossintético C3, crescimento inicial lento e largo espaçamento entre as linhas de cultivo (Freitas et al., 2002).

Recentemente, a expansão da área de cultivo do algodoeiro em safrinha parece ter aumentado a importância do controle adequado de plantas daninhas, especialmente no início do ciclo. Para o algodoeiro cultivado em segunda safra, principalmente em população adensada, o período anterior à interferência (PAI) passa de 17 dias após a emergência (DAE) para 4 DAE, em comparação com o algodoeiro cultivado em espaçamento convencional na safra (Raimondi, 2012). As perdas pela matocompetição no algodoeiro podem chegar a $90 \%$, quando não adotada nenhuma prática de manejo e havendo convivência das plantas daninhas desde o início do ciclo (Freitas et al., 2006).
Em função desta sensibilidade do algodoeiro à convivência com as plantas daninhas, torna-se imprescindível que métodos de controle visando à eliminação ou redução da interferência sejam empregados durante o ciclo da cultura, pois a não adoção de ações com esta finalidade acaba por reduzir a produtividade e a qualidade da fibra produzida. Dentre os diversos métodos de controle, o mais utilizado é o controle químico, que consiste no uso de herbicidas seletivos para a cultura (Braz et al., 2012). Entretanto, na maioria dos trabalhos realizados, os efeitos de seletividade e matointerferência não estão separados, podendo levar a erros de interpretação.

A seletividade de herbicidas aplicados em pré-emergência no algodoeiro ainda é pouco estudada. Muitos são os fatores que podem influenciar na seletividade, tais como, clima, textura e compactação do solo, dose e associação entre herbicidas. A mistura de herbicidas pode ser um valioso instrumento para o controle de plantas daninhas. A seletividade dos herbicidas pode ser afetada pelo uso de duas ou mais moléculas, pois uma mistura que apresentar sinergismo no controle pode não ser viável se acarretarem quedas de produtividade.

O objetivo deste trabalho foi avaliar a seletividade de herbicidas aplicados em préemergência, isolados ou em misturas, para diferentes variedades de algodoeiro.

\section{Material e Métodos}

Foram conduzidos dois experimentos no distrito de Iguatemi, município de Maringá - PR durante o período de janeiro a agosto de 2007, na Fazenda Experimental de Iguatemi (FEI) pertencente à Universidade Estadual de Maringá (UEM). As coordenadas geográficas de localização de ambos os experimentos foram latitude $23^{\circ} 25^{\prime} 56,70^{\prime \prime} \mathrm{S}$ e longitude $51^{\circ} 57^{\prime} 21,40^{\prime \prime} \mathrm{W}$, à 542 metros de altitude.

$\mathrm{O}$ solo da área experimental apresentava $280 \mathrm{~g} \mathrm{~kg}^{-1}$ de argila, $30 \mathrm{~g} \mathrm{~kg}^{-1}$ de silte, $690 \mathrm{~g} \mathrm{~kg}^{-1}$ de areia (Classe textural 
franco-argilo-arenoso), 16,04 $\mathrm{g} \mathrm{dm}^{-3}$ de matéria orgânica, pH em água igual a 6,0, sendo classificado como Argissolo Vermelho distrófico.

Segundo a classificação de Köeppen, o clima onde se localiza a área experimental é classificado como subtropical com chuvas de verão e invernos secos, Cfa. A temperatura média anual é de $21,69^{\circ} \mathrm{C}$, sendo $36,82^{\circ} \mathrm{C}$ a média das temperaturas máximas do mês mais quente, e $10,98^{\circ} \mathrm{C}$ a média das temperaturas mínimas do mês mais frio. Na Figura 1 estão representados os valores de temperatura máxima e mínima, além da precipitação, ambos diários, ocorridos durante o ciclo da cultura.

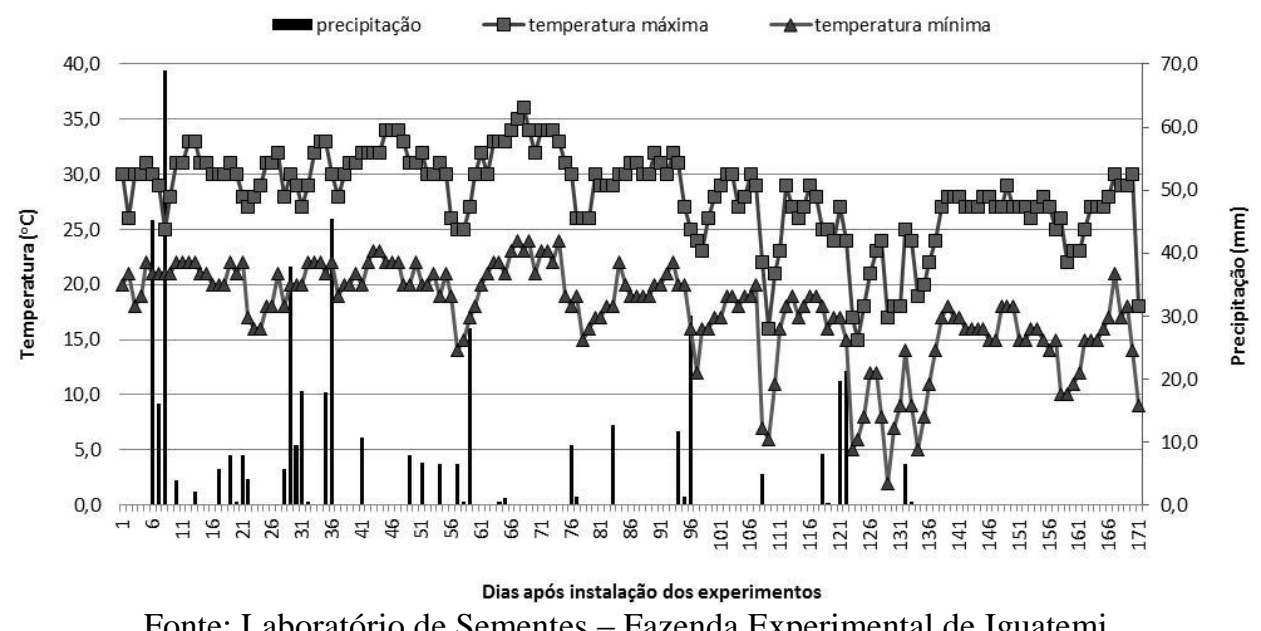

Fonte: Laboratório de Sementes - Fazenda Experimental de Iguatemi.

Figura 1. Temperatura máxima, mínima e precipitação diária durante o período de condução dos experimentos com a cultura do algodoeiro. Maringá, PR - 2007.

Antes da instalação do experimento, o solo foi revolvido por duas vezes, sendo a primeira com grade aradora e a segunda com grade niveladora, visando à descompactação e maior uniformização do perfil do solo na área experimental, além de eliminar todas as plantas daninhas emergidas. A semeadura da cultura do algodoeiro foi realizada em 22 de janeiro de 2007, utilizando uma semeadora com quatro linhas de semeadura, espaçadas $0,90 \mathrm{~m}$. A densidade de semeadura foi de 10 a 12 sementes por metro linear, sendo estas semeadas a uma profundidade aproximada de $2,0 \mathrm{~cm}$. A adubação utilizada no sulco de semeadura foi de $300 \mathrm{~kg} \mathrm{ha}^{-1}$ da fórmula comercial 04-20-20. Os dois experimentos foram conduzidos simultaneamente, avaliandose em cada um destes uma variedade de algodoeiro. As sementes utilizadas no primeiro experimento foram da variedade Delta-Opal e no segundo da variedade FMT-701.
Os tratamentos avaliados foram idênticos para ambos os experimentos, totalizando 15 herbicidas aplicados isolados ou em misturas em pré-emergência do algodoeiro (Tabela 1). O delineamento experimental utilizado foi o de blocos ao acaso, em esquema de parcelas subdivididas (15 x 2) com quatro repetições. O fator herbicida (15 tratamentos) foi alocado nas parcelas e o fator ausência e presença dos herbicidas (2 tratamentos) foi alocado nas subparcelas. Portanto, cada parcela foi composta por uma subparcela que recebeu a aplicação dos tratamentos herbicidas e por uma testemunha absoluta, que não recebeu nenhum tratamento herbicida. A adoção deste arranjo experimental permite a comparação (desdobramento) dos tratamentos herbicidas com as testemunhas que foram instaladas dentro de uma mesma parcela, o que consequentemente minimiza a variabilidade da área e o erro experimental com eficiência, o 
que é fundamental para experimentos de seletividade que avaliam um grande número de tratamentos.

Tabela 1. Tratamentos avaliados nos experimentos de seletividade de herbicidas aplicados em pré-emergência para diferentes variedades de algodoeiro. Maringá-PR - 2007.

\begin{tabular}{lc}
\hline \multicolumn{1}{c}{ Tratamento } & Dose (kg i.a. ha- $\left.\mathrm{h}^{-1}\right)$ \\
\hline Alachlor & 1,200 \\
S-metolachlor & 0,672 \\
Diuron & 0,900 \\
Diuron & 1,200 \\
Prometryn & 0,900 \\
Prometryn & 1,200 \\
Oxyfluorfen & 0,192 \\
Alachlor + diuron & $1,200+1,200$ \\
Alachlor + prometryn & $1,200+0,900$ \\
Alachlor + prometryn & $1,200+1,200$ \\
S-metolachlor + diuron & $0,672+1,200$ \\
S-metolachlor + prometryn & $0,672+0,900$ \\
S-metolachlor + prometryn & $0,672+1,200$ \\
Oxyfluorfen + diuron & $0,192+1,200$ \\
Oxyfluorfen + prometryn & $0,192+0,900$ \\
\hline
\end{tabular}

A unidade experimental compreendeu quatro linhas de plantio espaçadas entre si de $0,90 \mathrm{~m}$, com $5,00 \mathrm{~m}$ de comprimento, compreendendo uma área total de $18,00 \mathrm{~m}^{2}$ por parcela. A aplicação ocorreu no dia seguinte à semeadura, sendo em pré-emergência total (da cultura e das plantas daninhas). As condições de aplicação do primeiro experimento (DeltaOpal) foram: solo úmido; temperatura de $30^{\circ} \mathrm{C}$; umidade relativa do ar de 62\%; velocidade do vento de $1,0 \mathrm{~km} \mathrm{~h}^{-1}$ e céu parcialmente nublado. No momento da aplicação do segundo experimento (FMT-701), as condições de aplicação eram: solo úmido; temperatura de $26^{\circ} \mathrm{C}$; umidade relativa do ar de $69 \%$; velocidade do vento de $1,0 \mathrm{~km} \mathrm{~h}^{-1}$ e céu limpo. As aplicações foram realizadas com um pulverizador costal de pressão constante à base de $\mathrm{CO}_{2}$ (35 $\mathrm{lb} \mathrm{pol}^{-2}$ ) equipado com 5 bicos XR 110.02 , espaçados em $0,5 \mathrm{~m}$, proporcionando uma vazão de $200 \mathrm{~L} \mathrm{ha}^{-1}$ de calda.

Todas as parcelas foram mantidas livres da presença de plantas daninhas durante todo o ciclo da cultura por meio de capinas manuais, evitando desta maneira que a interferência da comunidade infestante interferisse nos resultados de seletividade dos herbicidas. As práticas culturais necessárias para a condução da lavoura, tais como o tratamento de sementes, controle de pragas e doenças, cobertura nutricional, utilização de regulador de crescimento, desfolhantes, entre outros, foram empregados de igual forma para todas as parcelas, de forma que a única variável fosse o tratamento herbicida avaliado.

Para avaliar o efeito dos herbicidas sobre a cultura foram realizadas avaliações de fitointoxicação aos 14, 21, 29 e 49 dias após a aplicação dos herbicidas (DAA). Para estas avaliações visuais, foram atribuídas notas conforme a escala proposta pela EWRC (1964) onde as notas variam entre $1=$ ausência de sintomas e $9=$ morte das plantas. Além disso, aos 21, 36 e 66 DAA, foram realizadas avaliações de altura das plantas de algodoeiro, medindo-se a altura da planta desde o nível do solo até a inserção da última folha completamente expandida. Para determinar a produtividade de cada tratamento, procedeu-se à colheita da área útil de cada unidade experimental, e os valores foram apresentados em rendimento de algodão em caroço em quilogramas por hectare, uniformizando a umidade para $13 \%$.

Os resultados obtidos no experimento foram submetidos à análise de variância, posteriormente a comparação do desdobramento do fator ausência e presença do tratamento herbicida foi comparado pelo teste F a $10 \%$ de probabilidade $(p<0,10)$. O desdobramento parcial da interação se justifica pelo objetivo do experimento, que é a avaliação da seletividade dos tratamentos herbicidas, já que um tratamento pode ser considerado seletivo quando o rendimento e a qualidade do produto colhido forem semelhantes a testemunha sem herbicida.

\section{Resultados e Discussão}

As avaliações visuais de fitointoxicação servem como importante parâmetro para 
verificar o potencial de utilização de determinado herbicida em uma cultura. Apesar disto, para fins conclusivos, somente esta avaliação não traz uma informação precisa sobre a seletividade apresentada por um herbicida, pois há casos em que o produto causa elevadas injúrias, mas não compromete a produtividade da cultura, ou ainda, aqueles em que o herbicida não provoca sintomas visuais de intoxicação nas plantas, mas há reduções no rendimento final (Barroso et al., 2012). Na Figura 2 estão apresentados os resultados das avaliações de fitointoxicação (14 DAA) de ambos os experimentos em função da aplicação dos herbicidas em pré-emergência do algodoeiro.

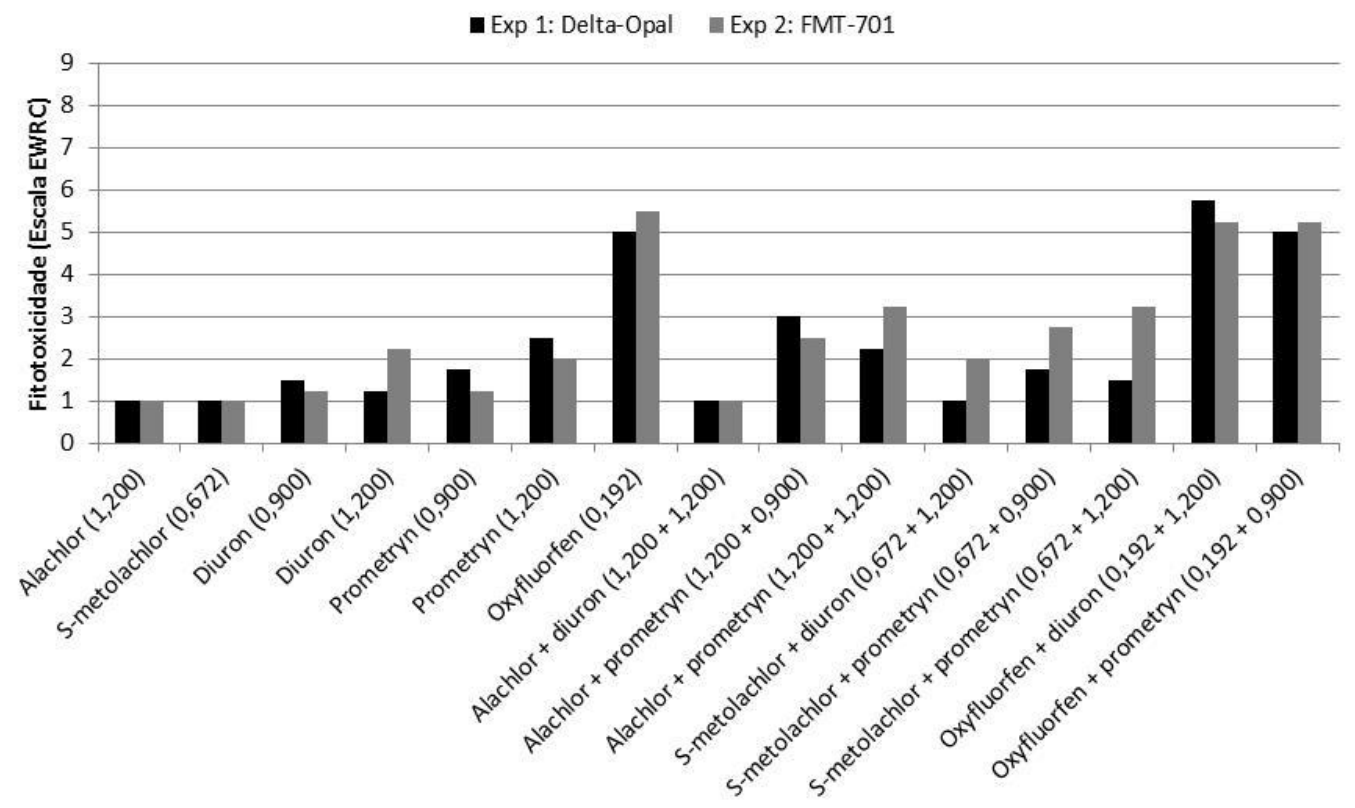

Figura 2. Notas de fitointoxicação (Escala EWRC) em duas variedades de algodoeiro aos 14 dias após tratamento com herbicidas (Doses em kg i.a. ha-1) em pré-emergência. Maringá, PR - 2007.

Os tratamentos que provocaram maiores sintomas de fitointoxicação foram os compostos pelo herbicida oxyfluorfen, seja este aplicado isolado ou em mistura com os herbicidas diuron e/ou prometryn. Os sintomas típicos observados nestes tratamentos foram necrose nos cotilédones de algumas plântulas das unidades experimentais, sem, contudo, haver morte das mesmas. As duas variedades de algodoeiro apresentaram comportamento semelhante para estes tratamentos, verificandose notas de fitointoxicação próximas. Os resultados observados no presente trabalho corroboram com dados já descritos na literatura, nos quais o oxyfluorfen aplicado em pré-emergência do algodoeiro provocou elevados sintomas de injúrias nas primeiras avaliações de fitointoxicação (Inoue et al., 2013).

Comparando a fitointoxicação observada em ambos os experimentos provocada pelos tratamentos com herbicidas isolados aos que continham misturas em tanque de produto, verifica-se que, excluindo $o$ oxyfluorfen, em todos os demais tratamentos verificaram-se injúrias mais acentuadas com a utilização de dois herbicidas associados (Figura 2). Em geral, quando se procede à associação de herbicidas, este comportamento é esperado, pois a planta terá a necessidade de se desintoxicar de mais de um princípio ativo diferente, e por vezes, os produtos associados apresentam mecanismos de ação distintos (Snipes \& Seifert, 2003; Dan et al., 2011). 
Outro herbicida que quando presente na composição do tratamento (isolado ou em mistura) causou maiores injúrias foi prometryn, observando-se leve necrose nas folhas verdadeiras e clorose do tecido vegetal presente nos cotilédones. De forma geral, para ambos os experimentos, apesar de terem sido observados sintomas de fitointoxicação aos 14 DAA, nas avaliações realizadas posteriormente $(21,29 \mathrm{e}$ 49 DAA), não se constatou injúrias nas novas folhas emergidas em nenhum dos tratamentos herbicidas avaliados (Dados não apresentados), mostrando que ambas as variedades, DeltaOpal e FMT-701, apresentaram a capacidade de se recuperar no decorrer do ciclo. No entanto, em condições de casa-de-vegetação, Yamashita et al. (2008) verificaram danos ao algodoeiro até 35 DAA, após a utilização do oxyfluorfen $\left(0,180 \quad \mathrm{~kg}^{\mathrm{h}} \mathrm{ha}^{-1}\right)$ em plantas cultivadas em substrato.

Nas três avaliações de altura realizadas, nota-se que as variedades estudadas apresentaram respostas diferenciadas em relação aos herbicidas testados. No experimento com a variedade Delta-Opal, em avaliação realizada aos 21 DAA, os tratamentos contendo alachlor aplicado isolado ou em associação com prometryn (em ambas as doses) reduziram o porte da planta (Tabela 2). O trabalho de Guimarães et al. (2007) confirma este resultado, visto que encontraram redução no porte da planta ao utilizar o herbicida alachlor, nas doses de 3,01 e 2,88 $\mathrm{kg} \mathrm{ha}^{-1}$.

Outros tratamentos que afetaram o desenvolvimento inicial do algodoeiro foram as misturas s-metolachlor + prometryn, em ambas as doses, além do herbicida oxyfluorfen aplicado isoladamente. Aos 36 DAA observase que os tratamentos contendo alachlor aplicado isolado ou em combinação com prometryn (em ambas as doses) não demonstraram recuperação na altura das plantas, conforme observado nas avaliações aos 21 DAA. Os tratamentos com oxyfluorfen isolado, s-metolachlor isolado e s-metolachlor
+ prometryn $\left(0,672+1,200 \mathrm{~kg} \mathrm{ha}^{-1}\right)$ também afetaram a altura das plantas nesta avaliação.

$\mathrm{Na}$ última avaliação realizada, aos 66 DAA, observa-se que todas estas diferenças entre as parcelas tratadas com herbicidas e suas respectivas testemunhas passaram a não existir, indicando a recuperação da cultura. Entretanto, o tratamento oxyfluorfen + prometryn, que até então não havia apresentado qualquer efeito significativo, passou a apresentar menor porte da planta.

Para o experimento com a variedade FMT-701, os herbicidas que promoveram menor altura das plantas aos 21 DAA foram: diuron $\left(0,900 \mathrm{~kg} \mathrm{ha}^{-1}\right)$; alachlor + diuron; smetolachlor + diuron; s-metolachlor + prometryn $\left(0,672+0,900 \mathrm{~kg} \mathrm{ha}^{-1}\right) \mathrm{e}$ oxyfluorfen + diuron. Aos 36 DAA os tratamentos com oxyfluorfen aplicado isoladamente, diuron $\left(0,900 \mathrm{~kg} \mathrm{ha}{ }^{-1}\right) \quad \mathrm{e}$ oxyfluorfen + diuron continuaram apresentando altura significativamente inferior às suas respectivas testemunhas (Tabela 2). Em avaliação aos 66 DAA não foram observadas tais diferenças, porém o tratamento alachor + prometrine $\left(1,200+0,900 \mathrm{~kg} \mathrm{ha}^{-1}\right)$ acarretou em altura significativamente inferior à sua respectiva testemunha.

Entre os tratamentos que afetaram o crescimento inicial das duas variedades de algodoeiro, observa-se maior efeito para os que continham prometryn, seja este isolado ou em associação a outros herbicidas. No experimento 2, cultivado com a variedade FMT-701, verifica-se maior efeito negativo sobre a altura das plantas nas parcelas que receberam aplicação de tratamentos com o herbicida diuron (isolado ou em mistura). Observa-se ainda que esta redução na altura está relacionada principalmente à paralisação ou diminuição temporária na taxa de crescimento das plantas, já que as avaliações de altura realizadas mais tardiamente indicam que a cultura retomou o seu desenvolvimento normal. 
Tabela 2. Altura (cm) de plantas de algodoeiro, variedades Delta-Opal e FMT-701, aos 21, 36 e 66 dias após o tratamento com herbicidas em pré-emergência. Maringá, PR - 2007

\begin{tabular}{|c|c|c|c|c|c|c|c|c|c|c|c|c|c|}
\hline \multicolumn{14}{|c|}{ Variedade Delta-Opal } \\
\hline \multirow{2}{*}{ Tratamento } & \multirow{2}{*}{$\begin{array}{c}\text { Dose } \\
\left(\mathrm{kg} \mathrm{i.a.} \text { ha }^{-1}\right) \\
\end{array}$} & \multicolumn{4}{|c|}{$21 \mathrm{DAA}$} & \multicolumn{4}{|c|}{36 DAA } & \multicolumn{4}{|c|}{$66 \mathrm{DAA}$} \\
\hline & & \multicolumn{2}{|c|}{ TRAT } & \multicolumn{2}{|l|}{ TD } & \multicolumn{2}{|c|}{ TRAT } & \multicolumn{2}{|c|}{ TD } & \multicolumn{2}{|c|}{ TRAT } & \multicolumn{2}{|l|}{ TD } \\
\hline Alachlor & 1,200 & 12,23 & $\mathrm{~b}$ & 14,50 & a & 33,95 & $\mathrm{~b}$ & 39,28 & $\mathrm{a}$ & 65,03 & $\mathrm{a}$ & 62,48 & $\mathrm{a}$ \\
\hline S-metolachlor & 0,672 & 11,98 & $\mathrm{a}$ & 13,68 & $\mathrm{a}$ & 34,08 & $\mathrm{~b}$ & 39,18 & $\mathrm{a}$ & 59,10 & $\mathrm{a}$ & 61,45 & $\mathrm{a}$ \\
\hline Diuron & 0,900 & 14,28 & $\mathrm{a}$ & 13,78 & $\mathrm{a}$ & 41,78 & $\mathrm{a}$ & 36,90 & $\mathrm{~b}$ & 62,30 & $\mathrm{a}$ & 63,35 & $\mathrm{a}$ \\
\hline Diuron & 1,200 & 11,63 & $\mathrm{a}$ & 12,63 & $\mathrm{a}$ & 34,58 & $\mathrm{a}$ & 36,15 & $\mathrm{a}$ & 59,23 & $\mathrm{a}$ & 59,60 & a \\
\hline Prometryn & 0,900 & 13,33 & $\mathrm{a}$ & 13,40 & $\mathrm{a}$ & 38,75 & $\mathrm{a}$ & 36,90 & $\mathrm{a}$ & 62,60 & $\mathrm{a}$ & 57,48 & $\mathrm{a}$ \\
\hline Prometryn & 1,200 & 13,38 & $\mathrm{a}$ & 13,93 & $\mathrm{a}$ & 37,83 & $\mathrm{a}$ & 39,60 & $\mathrm{a}$ & 66,80 & $\mathrm{a}$ & 64,25 & $\mathrm{a}$ \\
\hline Oxyfluorfen & 0,192 & 12,43 & b & 14,68 & $\mathrm{a}$ & 34,80 & $\mathrm{~b}$ & 39,25 & $\mathrm{a}$ & 64,35 & $\mathrm{a}$ & 65,48 & $\mathrm{a}$ \\
\hline Alachlor + diuron & $1,200+1,200$ & 11,63 & $\mathrm{a}$ & 13,38 & $\mathrm{a}$ & 34,10 & $\mathrm{a}$ & 36,68 & a & 61,15 & $\mathrm{a}$ & 63,05 & $\mathrm{a}$ \\
\hline Alachlor + prometryn & $1,200+0,900$ & 10,45 & b & 14,65 & $\mathrm{a}$ & 31,80 & $\mathrm{~b}$ & 39,80 & a & 70,18 & $\mathrm{a}$ & 63,78 & b \\
\hline Alachlor + prometryn & $1,200+1,200$ & 10,85 & b & 13,50 & a & 33,85 & $\mathrm{~b}$ & 38,43 & a & 68,68 & $\mathrm{a}$ & 69,40 & $\mathrm{a}$ \\
\hline S-metolachlor + diuron & $0,672+1,200$ & 13,73 & a & 13,75 & a & 38,18 & a & 38,45 & $\mathrm{a}$ & 68,20 & $\mathrm{a}$ & 64,77 & $\mathrm{a}$ \\
\hline S-metolachlor + prometryn & $0,672+0,900$ & 11,23 & $b$ & 13,35 & $\mathrm{a}$ & 35,65 & $\mathrm{a}$ & 37,43 & $\mathrm{a}$ & 69,98 & $\mathrm{a}$ & 69,10 & $\mathrm{a}$ \\
\hline S-metolachlor + prometryn & $0,672+1,200$ & 12,43 & $\mathrm{~b}$ & 14,53 & $\mathrm{a}$ & 34,75 & $\mathrm{~b}$ & 39,85 & a & 65,78 & $\mathrm{a}$ & 64,30 & $\mathrm{a}$ \\
\hline Oxyfluorfen + diuron & $0,192+1,200$ & 14,78 & $\mathrm{a}$ & 14,25 & $\mathrm{a}$ & 37,80 & $\mathrm{a}$ & 38,13 & $\mathrm{a}$ & 67,68 & a & 69,23 & $\mathrm{a}$ \\
\hline Oxyfluorfen + prometryn & $0,192+0,900$ & 13,45 & $\mathrm{a}$ & 14,58 & $\mathrm{a}$ & 37,30 & $\mathrm{a}$ & 39,58 & $\mathrm{a}$ & 65,35 & $\mathrm{~b}$ & 73,65 & a \\
\hline $\mathrm{CV}(\%)$ & & 12,01 & & & & 9,43 & & & & 6,79 & & & \\
\hline DMS (Tukey, 10\%) & & 1,88 & & & & 4,15 & & & & 5,22 & & & \\
\hline \multicolumn{14}{|c|}{ Variedade FMT-701 } \\
\hline \multirow{2}{*}{ Tratamento } & Dose & \multicolumn{4}{|c|}{$21 \mathrm{DAA}$} & \multicolumn{4}{|c|}{36 DAA } & \multicolumn{4}{|c|}{66 DAA } \\
\hline & $\left(\mathrm{kg}\right.$ i.a. ha $\left.\mathrm{ha}^{-1}\right)$ & \multicolumn{2}{|c|}{ TRAT } & \multicolumn{2}{|l|}{ TD } & \multicolumn{2}{|c|}{ TRAT } & \multicolumn{2}{|l|}{ TD } & \multicolumn{2}{|c|}{ TRAT } & TD & \\
\hline Alachlor & 1,200 & 13,00 & $\mathrm{a}$ & 13,80 & $\mathrm{a}$ & 39,60 & $\mathrm{a}$ & 42,43 & $\mathrm{a}$ & 67,90 & $\mathrm{a}$ & 70,35 & $\mathrm{a}$ \\
\hline S-metolachlor & 0,672 & 12,73 & $\mathrm{a}$ & 12,93 & a & 42,35 & $\mathrm{a}$ & 40,28 & $\mathrm{a}$ & 80,23 & $\mathrm{a}$ & 76,25 & a \\
\hline Diuron & 0,900 & 12,73 & $\mathrm{~b}$ & 14,90 & $\mathrm{a}$ & 38,40 & $\mathrm{~b}$ & 42,93 & $\mathrm{a}$ & 73,48 & $\mathrm{a}$ & 75,20 & $\mathrm{a}$ \\
\hline Diuron & 1,200 & 12,83 & $\mathrm{a}$ & 13,08 & $\mathrm{a}$ & 39,25 & a & 39,43 & $\mathrm{a}$ & 79,43 & $\mathrm{a}$ & 76,25 & $\mathrm{a}$ \\
\hline Prometryn & 0,900 & 13,48 & $\mathrm{a}$ & 13,03 & $\mathrm{a}$ & 42,40 & a & 39,40 & $\mathrm{a}$ & 79,63 & $\mathrm{a}$ & 80,55 & $\mathrm{a}$ \\
\hline Prometryn & 1,200 & 14,48 & $\mathrm{a}$ & 13,23 & $\mathrm{a}$ & 40,98 & $\mathrm{a}$ & 41,33 & $\mathrm{a}$ & 81,08 & $\mathrm{a}$ & 79,68 & $\mathrm{a}$ \\
\hline Oxyfluorfen & 0,192 & 12,15 & $\mathrm{a}$ & 13,53 & a & 35,20 & $\mathrm{~b}$ & 41,33 & $\mathrm{a}$ & 75,25 & $\mathrm{a}$ & 80,33 & $\mathrm{a}$ \\
\hline Alachlor + diuron & $1,200+1,200$ & 11,85 & b & 13,88 & $\mathrm{a}$ & 40,88 & $\mathrm{a}$ & 41,90 & $\mathrm{a}$ & 77,40 & $\mathrm{a}$ & 77,88 & a \\
\hline Alachlor + prometryn & $1,200+0,900$ & 13,00 & $\mathrm{a}$ & 14,30 & a & 40,48 & a & 42,35 & $\mathrm{a}$ & 74,63 & $\mathrm{~b}$ & 83,10 & $\mathrm{a}$ \\
\hline Alachlor + prometryn & $1,200+1,200$ & 13,30 & $\mathrm{a}$ & 13,13 & $\mathrm{a}$ & 39,93 & $\mathrm{a}$ & 38,35 & $\mathrm{a}$ & 78,13 & $\mathrm{a}$ & 75,10 & $\mathrm{a}$ \\
\hline S-metolachlor + diuron & $0,672+1,200$ & 11,00 & $\mathrm{~b}$ & 13,05 & $\mathrm{a}$ & 35,70 & a & 37,93 & $\mathrm{a}$ & 74,68 & $\mathrm{a}$ & 75,45 & a \\
\hline S-metolachlor + prometryn & $0,672+0,900$ & 12,23 & $\mathrm{~b}$ & 14,33 & a & 41,18 & a & 42,30 & $\mathrm{a}$ & 76,23 & a & 75,43 & a \\
\hline S-metolachlor + prometryn & $0,672+1,200$ & 11,03 & $\mathrm{a}$ & 12,00 & a & 34,35 & a & 37,43 & $\mathrm{a}$ & 73,63 & a & 72,15 & $\mathrm{a}$ \\
\hline Oxyfluorfen + diuron & $0,192+1,200$ & 10,93 & $\mathrm{~b}$ & 12,73 & $\mathrm{a}$ & 36,40 & $\mathrm{~b}$ & 40,55 & $\mathrm{a}$ & 69,60 & $\mathrm{a}$ & 72,15 & $\mathrm{a}$ \\
\hline Oxyfluorfen + prometryn & $0,192+0,900$ & 11,55 & $\mathrm{a}$ & 12,58 & $\mathrm{a}$ & 38,00 & $\mathrm{a}$ & 38,90 & $\mathrm{a}$ & 75,48 & $\mathrm{a}$ & 76,70 & $\underline{a}$ \\
\hline $\mathrm{CV}(\%)$ & & 9,61 & & & & 8,70 & & & & 5,85 & & & \\
\hline DMS (Tukey, 10\%) & & 1,47 & & & & 4,10 & & & & 5,27 & & & \\
\hline
\end{tabular}

TRAT: Tratamento - TD: Testemunha dupla adjacente. Médias seguidas das mesmas letras em cada linha não diferem entre si pelo teste $\mathrm{F}$ ( $10 \%$ de probabilidade)

Os resultados referentes ao rendimento de algodão em caroço em função da aplicação dos diferentes tratamentos herbicidas na préemergência do algodoeiro em ambos os experimentos estão apresentados na Tabela 3 . Nos dois experimentos, o único tratamento que comprometeu a produtividade do algodoeiro foi o composto pela mistura entre oxyfluorfen + prometryn $\left(0,192+0,900 \mathrm{~kg} \mathrm{ha}^{-1}\right)$ aplicado em pré-emergência. A redução na produtividade para ambas as variedades em relação à respectiva testemunha deste tratamento foi de 8,80 e $7,15 \%$ respectivamente para Delta-Opal e FMT-701.

Em trabalho semelhante, onde se utilizou a variedade FMT-701, verificou-se que o tratamento com associação de oxyfluorfen e prometryn, mesmo aplicado em doses maiores, apresentou seletividade para o algodoeiro, divergindo dos resultados obtidos no presente trabalho (Inoue et al., 2013). Um fato que pode explicar essa seletividade diferencial em ambos 
os trabalhos são as diferenças na textura e teor de matéria orgânica (M.O.) no solo, sendo que no trabalho descrito na literatura, ambas as características permitiam a utilização de maiores dosagens (teores de argila e M.O. maiores) sem comprometer o rendimento do algodoeiro.

Tabela 3. Produtividade de algodão em caroço $\left(\mathrm{kg} \mathrm{ha}^{-1}\right)$ das variedades Delta-Opal e FMT-701 após a aplicação de herbicidas em pré-emergência. Maringá, PR - 2007

\begin{tabular}{|c|c|c|c|c|c|c|c|c|c|}
\hline \multirow{2}{*}{ Tratamento } & \multirow{2}{*}{$\begin{array}{c}\text { Dose } \\
\left(\mathrm{kg} \mathrm{i.a.} \mathrm{ha}^{-1}\right)\end{array}$} & \multicolumn{4}{|c|}{ Delta-Opal } & \multicolumn{4}{|c|}{ FMT-701 } \\
\hline & & TRAT & & TD & & TRAT & & TD & \\
\hline Alachlor & 1,200 & 3211,34 & a & 3258,99 & a & 4022,57 & $\mathrm{a}$ & 4270,76 & $\mathrm{a}$ \\
\hline S-metolachlor & 0,672 & 3218,39 & $\mathrm{a}$ & 3320,92 & $\mathrm{a}$ & 4857,34 & $\mathrm{a}$ & 4686,89 & $\mathrm{a}$ \\
\hline Diuron & 0,900 & 3390,71 & $\mathrm{a}$ & 3332,29 & $\mathrm{a}$ & 4428,50 & $\mathrm{a}$ & 4616,93 & $\mathrm{a}$ \\
\hline Diuron & 1,200 & 2840,86 & a & 3172,57 & $\mathrm{a}$ & 4601,58 & $\mathrm{a}$ & 4859,07 & $\mathrm{a}$ \\
\hline Prometryn & 0,900 & 3057,00 & a & 2983,89 & a & 4629,92 & $\mathrm{a}$ & 4592,98 & a \\
\hline Prometryn & 1,200 & 3612,86 & $\mathrm{a}$ & 3349,03 & $\mathrm{a}$ & 5040,13 & $\mathrm{a}$ & 4753,43 & $\mathrm{a}$ \\
\hline Oxyfluorfen & 0,192 & 3057,00 & $\mathrm{a}$ & 3269,44 & $\mathrm{a}$ & 4515,65 & $\mathrm{a}$ & 4604,92 & $\mathrm{a}$ \\
\hline Alachlor + diuron & $1,200+1,200$ & 2920,82 & $\mathrm{a}$ & 3196,01 & $\mathrm{a}$ & 5136,02 & $\mathrm{a}$ & 4976,93 & $\mathrm{a}$ \\
\hline Alachlor + prometryn & $1,200+0,900$ & 3419,02 & $\mathrm{a}$ & 3379,93 & $\mathrm{a}$ & 4794,97 & $\mathrm{a}$ & 4881,10 & $\mathrm{a}$ \\
\hline Alachlor + prometryn & $1,200+1,200$ & 3547,53 & a & 3587,25 & $\mathrm{a}$ & 4957,05 & $\mathrm{a}$ & 4744,28 & a \\
\hline S-metolachlor + diuron & $0,672+1,200$ & 3435,59 & a & 3345,99 & $\mathrm{a}$ & 4460,63 & $\mathrm{a}$ & 4589,11 & a \\
\hline S-metolachlor + prometryn & $0,672+0,900$ & 3370,82 & a & 3463,44 & a & 4701,35 & $\mathrm{a}$ & 4478,06 & $\mathrm{a}$ \\
\hline S-metolachlor + prometryn & $0,672+1,200$ & 3416,58 & a & 3509,81 & $\mathrm{a}$ & 4146,71 & $\mathrm{a}$ & 4175,68 & $\mathrm{a}$ \\
\hline Oxyfluorfen + diuron & $0,192+1,200$ & 3699,07 & a & 3808,03 & $\mathrm{a}$ & 4087,01 & $\mathrm{a}$ & 4232,29 & $\mathrm{a}$ \\
\hline Oxyfluorfen + prometryn & $0,192+0,900$ & 3589,38 & $\mathrm{~b}$ & 3935,79 & $\mathrm{a}$ & 4181,98 & $\mathrm{~b}$ & 4504,09 & $\mathrm{a}$ \\
\hline $\mathrm{CV}(\%)$ & & & 5 , & & & & & 8,45 & \\
\hline DMS (Tukey, 10\%) & & & & & & & & 36,01 & \\
\hline
\end{tabular}

TRAT: Tratamento - TD: Testemunha dupla adjacente. Para cada variedade, médias seguidas das mesmas letras em cada linha não diferem entre si pelo teste $\mathrm{F}$ (10\% de probabilidade)

Os demais tratamentos avaliados apresentaram-se seletivos para ambas as variedades de algodoeiro, corroborando com dados já descritos na literatura, tanto para os herbicidas avaliados isoladamente quanto para as misturas em tanque (Dan et al., 2011; Barroso et al., 2012). Os resultados encontrados no presente trabalho consistem em importante informação visando à criação de novas ferramentas no manejo de plantas daninhas do algodoeiro, pois permite que a interferência inicial seja reduzida, pela ação em pré-emergência destes herbicidas, além de proporcionar a rotação de mecanismos de ação e utilização de associações em tanque, o que consiste em prática importante visando à prevenção da resistência de plantas daninhas a herbicidas (López-Ovejero et al., 2006).

\section{Conclusões}

As plantas das duas variedades que apresentaram redução em seu porte no início do desenvolvimento se recuperaram ao longo do ciclo da cultura, independente do tratamento herbicida recebido.

Nas condições em que se conduziu a presente pesquisa conclui-se que a associação de oxyfluorfen + prometryn na dose de $0,192+$ $0,900 \mathrm{~kg} \mathrm{ha}^{-1}$ não foi seletiva ao algodoeiro para as variedades Delta-Opal e FMT-701. Em contrapartida, todos os demais tratamentos foram seletivos ao algodoeiro, trazendo novas alternativas para o manejo de plantas daninhas nesta cultura.

\section{Referências}

BARROSO, A.L.L. et al. Selectivity of preemergence herbicide treatments for cotton crop. Bioscience Journal, v.28, n.5, p.762769, 2012.

BRAZ, G.B.P. et al. Seletividade de amonioglufosinate isolado e em mistura com pyrithiobac-sodium em algodoeiro transgênico 
LL $^{\circledR}$. Planta Daninha, v.30, n.4, p.853-860, 2012.

CONSTANTIN, J. et al. Efeito de subdoses de 2,4-D na produtividade do algodão $\mathrm{e}$ suscetibilidade da cultura em função de seu estádio de desenvolvimento. Engenharia Agrícola, v.27, n.esp., p.24-29, 2007.

DAN, H.A. et al. Seletividade de clomazone isolado ou em mistura para a cultura do algodoeiro. Planta Daninha, v.29, n.3, p.601607, 2011.

EWRC (European Weed Researsh Council). Report of 3rd and 4th meetings of EWRC Committee of Methods in Weed Research. Weed Research, v.4, p.88, 1964.

FREITAS, R.S. et al. Interferência de plantas daninhas na cultura de algodão em sistema de plantio direto. Planta Daninha, v.20, n.2, p.197-205, 2002.

FREITAS, R.S. et al. Manejo de plantas daninhas na cultura do algodoeiro com Smetolachlor e trifloxysulfuron-sodium em sistema de plantio convencional. Planta Daninha, v.24, n.2, p.311-318, 2006.

GUIMARÃES, S.C.; HRYCYK, M.F.; MENDONÇA, E.A.F. Efeito de fatores ambientais sobre a seletividade do alachlor ao algodoeiro. Planta Daninha, v.25, n.4, p.813821, 2007.

INOUE, M.H. et al. Seletividade de herbicidas aplicados em pré-emergência na cultura do algodão. Revista Ciência Agronômica, v.44, n.1, p.123-132, 2013.

LÓPEZ-OVEJERO, R.F. et al. Resistance and differential susceptibility of Bidens pilosa and $B$. subalternans biotypes to ALS-inhibiting herbicides. Scientia Agricola, v.63, n.2, p.139145, 2006.

RAIMONDI, M.A et al. Periods of weeds interference in cotton yield in the seeding densified "Off Season". Planta Daninha, v.32, n.3, p.521-532, 2014.
SNIPES, C.E., SEIFERT, S. Influence of malathion timing on cotton (Gossypium hirsutum) response to pyrithiobac. Weed Technology, v.17, n.2, p.266-268, 2003.

YAMASHITA, O.M. et al. Efeito de doses reduzidas de oxyfluorfen em cultivares de algodoeiro. Planta Daninha, v.26, n.4, p.917921, 2008. 